Research Article

Open Access

\author{
Güvenç Görgülü*
}

\title{
Experimental and theoretical study of a novel naphthoquinone Schiff base
}

https://doi.org/10.1515/chem-2018-0121

received July 23, 2018; accepted September 7, 2018.

\begin{abstract}
A novel Schiff base was synthesized and characterized by spectroscopic and theoretical methods. A potentially active agent 4-(2-hydroxy-5methylphenylimino)naphthalen-1(4H)-one (PINQ) was designed and synthesized. The synthesis was carried out by a condensation reaction of 1-4-naphthoquinone and 2-amino-4-methyl phenol. The spectral and structural properties of the PINQ molecule were investigated by elemental analysis, ${ }^{1} \mathrm{H}-$ and ${ }^{13} \mathrm{C}-\mathrm{NMR}$, FT-IR and Uv-vis spectroscopies. The energetic, atomic, electronic, molecular, vibrational and magnetic data were theoretically obtained using density functional theory (DFT) at B3LYP level with 6-311++G(d,p) basis set. Chemical shifts were calculated using gauge-invariant atomic orbital (GIAO) method. UV-vis spectrum for the title compound was also obtained by time-dependent density functional theory (TD-DFT). The theoretical and experimental results were compared and interpreted. The theoretical data obtained from ${ }^{1} \mathrm{H}$ - and ${ }^{13} \mathrm{C}$-NMR, FT-IR and Uv-vis spectroscopies were quite compatible with experimental ones.
\end{abstract}

Keywords: Schiff base; Naphthoquinone; DFT.

\section{Introduction}

Schiff bases are still having a great deal of attraction since they have been first synthesized in 1864 [1]. They have a broad area of application from pharmacology and medicine to industry. In medicine, Schiff bases have been used as antioxidant, anticancer, antiviral, antibacterial, antifungal and more [2-5] for decades besides they are being pioneers for many other chemical compounds $[6,7]$. Moreover, new infectious pathogens emerging and multidrug resistance is a challenging problem for medicine [8]. Using any biological enzyme as a pharmacological agent is not possible, for the time being, due to its delivery problems and instability in solution. Therefore, characteristically different new agents have been synthesized and characterized by the researchers $[9,10]$.

Containment of some specific substituent groups by Schiff bases may give rise to more stabilized and active structures which is essential for an active agent. Various Schiff bases are used as ligands getting into coordination with transition metal ions to form biologically active metal complexes. Any information, like biological half-life and many other pharmacodynamic and pharmacokinetic features which are received during characterization, should be considered and evaluated to understand the mechanism of action of Schiff bases [5]. In addition to the spectroscopic techniques, which are vital for characterization, 3D structural, angular, molecular, atomic, electronic, energetic, transitional, vibrational and magnetic information about the target molecule can be obtained by quantum chemical calculations. Better results have been acquired by the researchers since the experimental and theoretical data were combined. Density functional theory (DFT) has been a major quantum chemical method for the theoretical evaluation of basic, thermodynamic, electronic and other properties of interested compounds [11].

This study is designed to synthesize and enlighten a biologically active compound both experimentally and theoretically which is the first step to produce a pharmacologically active drug. For this purpose, a naphthoquinone molecule is reacted with a phenolic amine to obtain 4-(2-hydroxy-5-methylphenylimino) naphthalen-1(4H)-one (PINQ). The compound was then characterized by spectroscopic methods and DFT calculation. The theoretical data obtained here were compared with the experimental results.

*Corresponding author: Güvenç Görgülü, Mehmet Akif Ersoy University, Faculty of Education, Department of Science Education, Burdur, Turkey, E-mail: guvencgorgulu@mehmetakif.edu.tr 


\section{Experimental}

\subsection{Physical measurements}

All chemicals with the highest purity grade were purchased from commercial sources.

A Shimadzu IRPrestige-21 FTIR spectrophotometer in the range of $4000-400 \mathrm{~cm}^{-1}$ was used to obtain the IR spectrum of PINQ molecule in $\mathrm{KBr}$ pellets. For the ${ }^{1} \mathrm{H}$ - and ${ }^{13} \mathrm{C}$-NMR spectra, tetramethylsilane (TMS) were used as an internal standard and chloroform as the solvent. The record was taken on a JEOL NMR-400 MHz spectrometer. The elemental analysis was taken in LECO 932 CHNS equipment. Spectrophotmetric measurements were recorded by a PG T80+ double-beam spectrophotometer in dry ethanol. Melting point of the compound was measured using an Electrothermal model IA 9100.

\subsection{Synthesis of the Schiff Base (PINQ)}

For the synthesis of the compound PINQ as shown in Figure 1 , a solution of $1 \mathrm{mmol}, 0.123 \mathrm{~g}$ of 2-amino-4-methylphenol in $15 \mathrm{~mL}$ of absolute ethanol was added to a solution of $1 \mathrm{mmol}, 0.158 \mathrm{~g}$ 1,4-naphthoquinone in $15 \mathrm{~mL}$ of another absolute ethanol solution. The mixture was left stirring for the next $6 \mathrm{~h}$ and kept $12 \mathrm{~h}$ at $25^{\circ} \mathrm{C}$. The precipitation was filtered off and rinsed for multiple times with cold ethanol and put on $\mathrm{P}_{2} \mathrm{O}_{5}$ for drying. Dried compound obtained in the powder form is stable at room temperature.

Brown compound; yield: $71 \%$; m.p.: $255^{\circ} \mathrm{C}$ (decomposition). Anal. Calc. for $\mathrm{C}_{17} \mathrm{H}_{13} \mathrm{NO}_{2}$ : C, 77.55; $\mathrm{H}$, 4.98; N, 5.32\%; Found: C, 77.42; H, 4.83; N, 5.41\%. ${ }^{1} \mathrm{H}-\mathrm{NMR}$ $\left(\mathrm{CDCl}_{3}, \mathrm{ppm}\right)$ : Theo. Calc. $6.62(1 \mathrm{H}, \mathrm{O}-\mathrm{H}), 6.84-8.74(7 \mathrm{H}$, $\mathrm{Ar}-\mathrm{H}), 2.38$ (3H, - $\left.-22 \mathrm{H}_{3}\right), 7.80(1 \mathrm{H},-\mathrm{H} 25), 6.84$ (1H, -H26); Found: 6.19 (1H, O-H), 6.94-8.64 (m, 7H, Ar-H), 2.32 (s, 3H, $\left.-\mathrm{C}_{20 \mathrm{H}_{3}}\right), 7.54$ (d, 1H, -H25), 6.84 (d, 1H, -H26). ${ }^{13} \mathrm{C}-\mathrm{NMR}$ ( $\mathrm{CDCl}_{3}, \mathrm{ppm}$ ): Theo. Calc. 191.20 (C10), 159.34 (C7), 119.01158.94 (Ar), 139.07 (C9), 135.58 (C8), 20.64 (C20); Found: 183.98 (C10), 146.85 (C7), 104.11-145.28 (Ar), 124.43 (C9), 116.18 (C8), 20.72 (C20). FT-IR (KBr, cm $\left.{ }^{-1}\right)$ : Theo. Calc. 3471 b (O-H), $1679 \mathrm{~s}(\mathrm{C}=\mathrm{O}), 1608 \mathrm{~m}(\mathrm{C}=\mathrm{N}), 1490 \mathrm{~s}(\mathrm{C}-\mathrm{N}), 1291 \mathrm{~s}$ (C-O); Found: b (O-H), $1681 \mathrm{~s}(\mathrm{C}=\mathrm{O}), 1612 \mathrm{~m}(\mathrm{C}=\mathrm{N}), 1491$ s (C-N), 1296 s (C-O),(b, broad; s, strong; m, medium; w, weak). UV-Vis (ethanol solution, nm): Theo. Calc. 408, 439, 563; Found: 311, 405, 507.


Figure 1: Synthesis reaction of PINQ molecule.

\subsection{Computational Methods}

Calculations were executed with Gaussian 09 program [11]. Molecular and spectral visualizations were performed by GaussView 5.0.9 software package [13]. The optimization of the molecular geometry and the vibrational frequency of the PINQ molecule were calculated by using DFT/ B3LYP with 6-311++G(d,p) basis set in the gas phase. The UV-vis spectrum, the highest occupied molecular orbital (HOMO) and the lowest unoccupied molecular orbital (LUMO) energies of the compound were determined by TD-DFT/B3LYP/6-311++G(d,p). The Gauge-invariant atomic orbital (GIAO) method which provides an effective prediction of the theoretical chemical shift values [14], with $6-311++G(d, p)$ basis set was used for the calculation of ${ }^{1} \mathrm{H}$ - and ${ }^{13} \mathrm{C}$-NMR chemical shifts $[15,16]$.

The HOMO-LUMO energy (E) gap $\left(\Delta \mathrm{E}_{\text {gap }}\right)$ could be expressed as follows:

$$
\Delta \mathrm{E}_{\text {gap }}=\mathrm{E}(\text { LUMO })-\mathrm{E}(\mathrm{HOMO})
$$

Ethical approval: The conducted research is not related to either human or animal use.

\section{Results and Discussion}

The physical and spectral data are given in the experimental section. Elemental analysis is a powerful technique for the identification of newly synthesized compounds which is time and cost effective. The $\mathrm{C}, \mathrm{N}$ and $\mathrm{H}$ percentages obtained from elemental analysis are so close to the calculated values of these elements which indicate that the synthesized PINQ molecule is in a high purity. One more evidence for the purity is the decomposition of the PINQ molecule which starts at $255^{\circ} \mathrm{C}$ and completed in a few degrees. 
Table 1: Representative bond lengths, bond and dihedral angles of PINQ molecule.

\begin{tabular}{|c|c|c|c|c|c|}
\hline \multicolumn{2}{|c|}{ Bond lengths $(\AA)$} & \multicolumn{2}{|c|}{ Bond angles $\left(^{\circ}\right)$} & \multicolumn{2}{|l|}{ Dihedral angles $\left(^{\circ}\right)$} \\
\hline C8-C9 & 1.35 & C9-C10-011 & 121.14 & C7-N12-C13-C18 & 37.83 \\
\hline C9-C10 & 1.47 & $\mathrm{C} 10-\mathrm{C} 5-\mathrm{C} 4$ & 121.03 & C7-N12-C13-C14 & 149.30 \\
\hline C10-011 & 1.22 & $\mathrm{C} 15-\mathrm{C} 4-\mathrm{C} 7$ & 120.20 & C6-C5-C10-011 & 0.44 \\
\hline C1-C2 & 1.40 & C8-C7-N12 & 124.52 & C3-C4-C7-C8 & 178.99 \\
\hline C7-N12 & 1.30 & C7-N12-C13 & 126.72 & C3-C4-C7-N12 & 2.23 \\
\hline $\mathrm{N} 12-\mathrm{C} 13$ & 1.39 & C13-C14-019 & 120.09 & C8-C7-N12-C13 & 12.12 \\
\hline C14-019 & 1.35 & $\mathrm{~N} 12-\mathrm{C} 13-\mathrm{C} 14$ & 114.32 & N12-C13-C14-019 & 4.02 \\
\hline O19-H30 & 0.97 & C20-C17-C16 & 120.57 & & \\
\hline C17-C20 & 1.51 & & & & \\
\hline $\mathrm{C} 20-\mathrm{H} 31$ & 1.10 & & & & \\
\hline
\end{tabular}

\subsection{Molecular Geometry}

The structural optimization geometry of the interested molecule yields valuable information including bond lengths, bond angles and dihedral angles (Table 1). The optimized chemical structure for PINQ is shown in Figure 2. The phenol and naphthoquinone parts of the molecule are not in the same plane but, N12 atom seems to be in the same planar geometry by each moiety. The dihedral angle C7-N12-C13-C18 with $37.83^{\circ}$ points out a deviation of the phenol moiety from the naphthoquinone part of the PINQ molecule.

The Mulliken atomic charges of PINQ molecule were also obtained with the calculation of geometry optimization. The highest positive charge was seen on H30 phenolic proton (0.292113) and the highest negative charge was shared between $\mathrm{C} 10$ of the carbonyl group $(-0.627888)$ and $\mathrm{C} 14$ atom of the phenolic group $(-0.628825)$ as expected. The positive charges around H30 can be explained by the electronegativity of $\mathrm{O} 19$ atom.

\section{$3.2{ }^{1} \mathrm{H}$ - and ${ }^{13} \mathrm{C}-\mathrm{NMR}$ Spectra}

The ${ }^{1} \mathrm{H}$ - and ${ }^{13} \mathrm{C}$-NMR spectra obtained from experimental and theoretical study were compared in Figures 3 and 4. Both spectra were combined on the same scale for an easier comparison. Experimental ${ }^{1} \mathrm{H}-\mathrm{NMR}$ chemical shifts were, expressed in ppm, dispersed through the spectrum starting form tetramethylsilane as the internal standard. The theoretical calculations are used to confirm the data obtained by the experiments reported herein. PINQ molecule was optimized (Figure 2) and the output file of optimization was used for the GIAO method calculations.

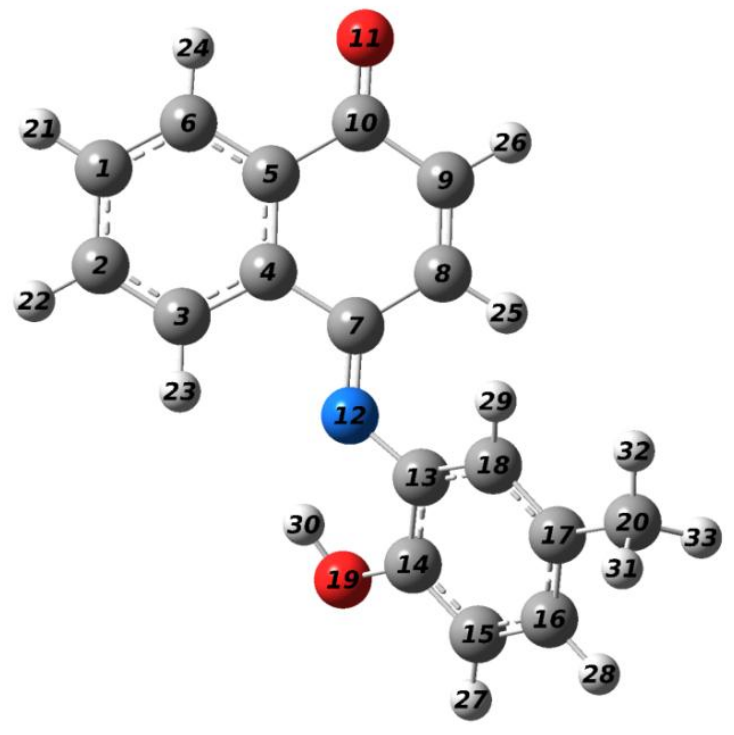

Figure 2: Optimized and numbered structure of PINQ molecule.

As seen from the Figure 3, peaks belong to methyl hydrogens splitted into three between 2.02 and $2.45 \mathrm{ppm}$ in the theoretical spectrum were seen as one peak in the experimental spectrum since the methyl protons have the same chemical environment leading their overlaps. The experimental methyl proton chemical shift values were stated as the average of three protons at $2.38 \mathrm{ppm}$. The peak seen at $6.19 \mathrm{ppm}$ was assigned to deuterium exchangeable proton of the phenolic $-\mathrm{OH}$ group. The chemical shift value of this proton was $6.62 \mathrm{ppm}$ in the calculated spectrum. The multiple peaks attributed to the aromatic protons between 6.94 and $8.64 \mathrm{ppm}$ were calculated approximately at the same range between 6.84 and $8.74 \mathrm{ppm}$. The peaks ascribed to the protons numbered 



Figure 3: Experimental (above) and theoretical (below) ${ }^{1} \mathrm{H}-\mathrm{NMR}$ spectra of the PINQ.

as 25 and 26 bound to aliphatic carbons were appeared at 7.54 and $6.84 \mathrm{ppm}$, respectively. These peaks of aliphatic protons were corresponded to the peaks at 7.80 and 6.84 ppm in the theoretical spectrum.

${ }^{13} \mathrm{C}$-NMR spectrum showed a chemical shift at 146.85 ppm assigned to the imine group revealing the formation of the PINQ molecule by the condensation reaction of 1,4-naphthoquinone and 2-amino-4-methylphenol. This chemical shift was observed at $159.34 \mathrm{ppm}$ in the theoretical spectrum. The peaks at 104.11-145.28 ppm range assigned to aromatic carbons were seen between 119.01$158.94 \mathrm{ppm}$ in the calculated spectrum. The calculated chemical shift belong to carbonyl carbon (C10) given at 191.20 was obtained at $183.98 \mathrm{ppm}$. In addition, the peak observed at $20.72 \mathrm{ppm}$ was attributed to the methyl carbon (C20) bounded to aromatic group. This chemical shift was calculated as $20.64 \mathrm{ppm}$ (Figure 4).

The ${ }^{1} \mathrm{H}$ - and ${ }^{13} \mathrm{C}$-NMR spectra have no unidentified peaks other than the expected ones (Figure 3 and 4). These clear spectra also reveal that the molecule is highly pure.

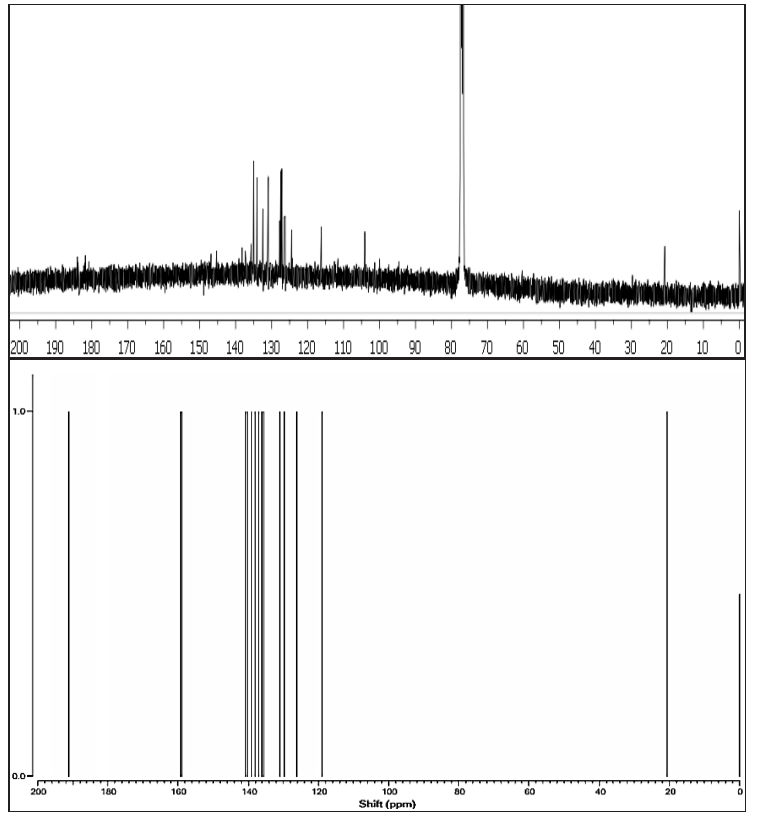

Figure 4: Experimental (above) and theoretical (below) ${ }^{13} \mathrm{C}-\mathrm{NMR}$ spectra of the PINQ.

\subsection{FT-IR Spectra}

The experimental spectrum of PINQ molecule was obtained between $400-4000 \mathrm{~cm}^{-1}$ and the calculations of vibrational frequencies were performed at the B3LYP level with $6-311++G(d, p)$ basis set (Figure 5). For precision, the theoretical data acquired from the frequencies below and above, respectively, $1700 \mathrm{~cm}^{-1}$ were scaled by the correction factors of 0.983 and 0.958 which was described elsewhere $[15,16]$.

As seen in Figure 5, the band at $1612 \mathrm{~cm}^{-1}$ corresponds to $v(\mathrm{C}=\mathrm{N})$ in PINQ molecule was calculated as $1608 \mathrm{~cm}^{-1}$. This stretching band is characteristic for imine group, revealing that the condensation reaction was completed and the desired molecule was formed. Phenolic $-\mathrm{OH}$ group showed a band at $3324 \mathrm{~cm}^{-1}$ which was observed at $3471 \mathrm{~cm}^{-1}$ on the theoretical spectrum. Observation of this band is the indication of the binding of phenolic moiety to 1,4-naphthoquinone molecule. Stretching vibration for the $\mathrm{C}=\mathrm{O}$ bond in the IR spectrum was recorded at $1681 \mathrm{~cm}^{-1}$. This band was appeared at $1679 \mathrm{~cm}^{-1}$ on the theoretical spectrum which is almost in the same frequency with experimental one. Finally, $v(\mathrm{C}-\mathrm{N})$ and $v(\mathrm{C}-\mathrm{O})$ were observed at $1491 \mathrm{~cm}^{-1}$ and $1296 \mathrm{~cm}^{-1}$ which were theoretically calculated as 1490 and $1291 \mathrm{~cm}^{-1}$, respectively. 
Table 2: The experimental and theoretical electronic transitions, excitation energies (eV), oscillator strengths (f) and major contributions of the PINQ molecule.

\begin{tabular}{lllllll}
\hline Transitions & \multicolumn{2}{l}{ Experimental } & \multicolumn{2}{l}{ Theoretical } \\
& \multicolumn{5}{c}{ B3LYP/6-311++G(d,p) } \\
\hline & $\lambda(\mathrm{nm})$ & $\mathrm{E}(\mathrm{eV})$ & $\lambda(\mathrm{nm})$ & $\mathrm{E}(\mathrm{eV})$ & $\mathbf{f}$ & Major contributions \\
$\mathrm{n} \rightarrow \pi^{\star}$ & 507 & 2.445 & 563 & 2.202 & 0.2743 & HOMO $\rightarrow$ LUMO (97\%) \\
$\pi \rightarrow \pi^{\star}$ (imine) & 405 & 3.061 & 439 & 2.824 & 0.0438 & HOMO-1 $\rightarrow$ LUMO (76\%) \\
$\pi \rightarrow \pi^{*}$ (benzene) & 311 & 3.987 & 408 & 3.039 & 0.0585 & HOMO-4 $\rightarrow$ LUMO (37\%) \\
& & & & & & HOMO-2 $\rightarrow$ LUMO (27\%) \\
& & & & & & HOMO-1 $\rightarrow$ LUMO (19\%) \\
\hline
\end{tabular}

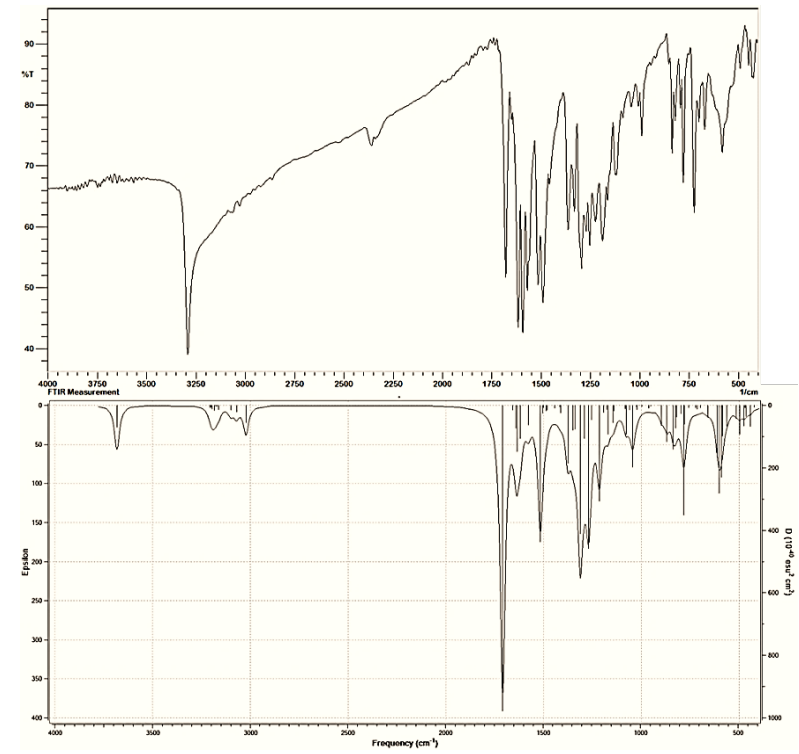

Figure 5: Experimental (above) and theoretical (below) FT-IR spectra of PINQ molecule.

\subsection{UV-vis Spectra}

The UV-vis spectrum of the PINQ molecule was recorded in $10^{-3} \mathrm{M}$ of ethanolic solution at room temperature. The electronic transitions were also theoretically calculated by using B3LYP/6-311++G(d,p) level and TD-DFT methods. The experimental and theoretical electronic transitions, excitation energies (eV), oscillator strengths (f) and major contributions of the PINQ are given in Table 2.

The measured and computed maximum UV-vis absorption wavelengths for PINQ are shown in Figure 6. The bands observed at 507, 405 and $311 \mathrm{~nm}$ in electronic spectrum of PINQ was calculated as 563, 439 and 408 nm. These three transitions, which are characteristic for aromatic imine molecules, were assigned to $n \rightarrow \pi^{\star}$, $\pi \rightarrow \pi^{\star}$ (imine) and $\pi \rightarrow \pi^{\star}$ (benzene), respectively. Electron transitions occurred between HOMO and LUMO have some



Figure 6: Experimental (above) and calculated (below) Uv-vis absorption spectra of the PINQ.

major and minor contributing orbitals. As seen from the theoretical absorption spectrum, the highest wavelength (563 nm) arises from HOMO to LUMO transition with $97 \%$ share. The electronic transition at $439 \mathrm{~nm}$ was from HOMO-1 to LUMO with $76 \%$ contribution. The major contributions for the transition at $408 \mathrm{~nm}$ were from HOMO-4 to LUMO with $37 \%$, HOMO-2 to LUMO with $27 \%$ and HOMO-1 to LUMO with $19 \%$. 


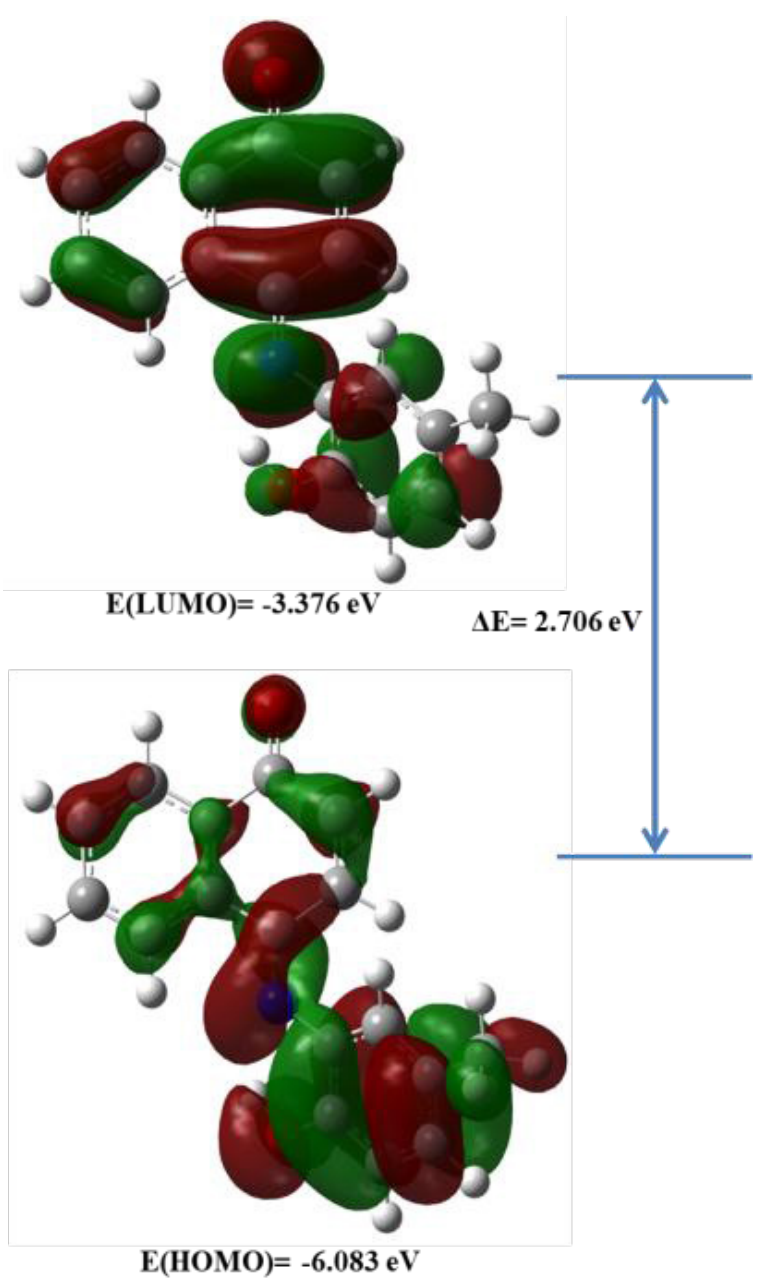

Figure 7: Energy levels and localization distribution of HOMO and LUMO molecular orbitals throughout the PINQ molecule as the representatives of frontiers.

The properties of the molecules such as the chemical reactivity, kinetic stability, polarizability, chemical hardness and softness, aromaticity and electronegativity can be determined by using this energy gap [19,20] between the HOMO and LUMO orbitals. The energy gap between HOMO and LUMO orbitals of PINQ molecule was calculated as $2.706 \mathrm{eV}$ (Figure 7). The gap value is inversely proportional with the softness and the reactivity of the molecule. For PINQ molecule, the gap value indicates a soft and reactive compound compared to similar naphthoquinone based molecules [21,22]. HOMO orbitals of PINQ were densely located on phenol rings and the LUMO were largely accumulated on naphthoquinone moiety.



Figure 8: Molecular electrostatic potential (MEP) of PINQ molecule in which electron-rich and poor regions are shown by red and blue colors, respectively.

\subsection{Molecular Electrostatic Potential (MEP)}

Molecular electrostatic potential of the PINQ molecule is shown in Figure 8. The colorized figure is a good indicator of inter- and intramolecular interactions and reactivity of the molecule [23]. The red color and blue color locates the electron-rich and poor regions, respectively. The carbonyl oxygen underlying the reddish area shows an electron rich part of the PINQ which is potentially the most aggressive region for a nucleophilic attack. On the contrary, the opposite site of the molecule has an electron poor region with a bluish color overlying a phenolic proton which may show an electrophilic behavior.

\section{Conclusion}

We herein reported the synthesis and spectral characterization of a novel imine molecule. The synthesis of PINQ was achieved by the condensation of a ketone and aromatic amine compounds. The spectral data revealed that, the reaction was completed and the target molecule was formed. Overall comparison of spectral data confirms a good correlation between the experimental and calculated results. The optimization of the PINQ molecule showed a non-planar molecular geometry with a torsion angle of $37.83^{\circ}$ between the naphthoquinone and phenolic moieties. The effective nucleophilic capacity of the carbonyl and phenolic oxygen of the molecule was brought out by Mulliken charges and the molecular electrostatic potential calculated. For the PINQ molecule, the gap value indicates a soft and reactive compound compared to similar naphthoquinone based molecules. 
Conflict of interest: Authors declare no conflict of interest.

\section{References}

[1] Tidwell T.T., Hugo (Ugo) Schiff, Schiff Bases and a century of $\beta$-Lactam synthesis, Angew. Chem. Int. Ed., 2008, 47(6), 10161020.

[2] Kemnitzer W., Sirisoma N., May C., Tseng B., Drewe J., Cai S.X., Discovery of 4-anilino- $\mathrm{N}$-methylthieneo[3,2-d]pyrimidines and 4-anilino-N-methylthieno[2,3-d]pyrimidines as potent apoptosis inducers, Bioorg. Med. Chem. Lett., 2009, 19, 3536-3540.

[3] Pavan K.K., Mohan S., Saravanan J., Vanitha P.K., Appalaraju N., Venkaeswara R.J., Synthesis and antimicrobial screening of some acid chloride derivatives of 2-amino- $\mathrm{N}$ - (3-chlorophenyl)5,6-dihydro-4H-cyclopenta[b]thiophene-3-carboxamide, Int. J. Chem. Sci., 2007, 5, 1284-1290.

[4] Katada J., lijima K., Muramatsu M., Takami M., Yasuda E., Hayashi M., et al., Cytotoxic effects of NSL-1406, a new thienopyrimidine derivative, on leukocytes and osteoclasts, Bioorg. Med. Chem. Lett., 1999, 9, 797-802.

[5] Brodowska K., todyga E.C., Schiff bases-interesting range of applications in various fields of science, Chemik., 2014, 68, 2, 129-134.

[6] Liu G., Cogan D., Ellman J.A., Catalytic asymmetric synthesis of tert-butanesulfinamide. Application to the asymmetric synthesis of amines, J. Am. Chem. Soc., 1997, 119(41), 9913-9914.

[7] Cimarelli C., Palmieri G., Asymmetric reduction of enantiopure imines with zinc borohydride: Stereoselective synthesis of chiral amines. Tetrahedron: Asymmetry, 2000, 11(12), 25552563.

[8] Biot C., François N., Maciejewski L., Brocard J., Poulain D., Synthesis and antifungal activity of a ferrocene-fluconazole analogue, Bioorg. Med. Chem. Lett., 2000, 10(8), 839-841.

[9] Gorgulu G., Dede B., Enzymatic activities of a novel dinuclear $\mathrm{Cu}(\mathrm{II})-\mathrm{Ni}(\mathrm{II})$ complex: design, synthesis and characterization, Fresen. Environ. Bull., 2018, 27(6), 3958-3964.

[10] Oturak H., Kinaytürk N.K., Şahin G., Structure and vibrational studies of \pm 1 -(1H-benzoimidazol-2-yl)ethanol, using DFT method, A. Phys. Pol. A., 2015, 128, B417-B421.

[11] Dede B., Özen N., Görgülü G., Synthesis, characterization, theoretical calculations and enzymatic activities of novel diiminedioxime ligand and its homodinuclear Cu(II) complex, J. Mol. Struct., 2018, 1163, 357-367.

[12] Frisch M.J., Trucks G.W., Schlegel H.B., Scuseria G.E., et al., Fox D.J. Gaussian, Inc., Wallingford CT, 2016.

[13] GaussView, Revision 5.0.9, Dennington R., Keith T.A., Millam J.M., Semichem Inc., Shawnee Mission, KS, 2009.

[14] Eryilmaz S., Akdemir N., Inkaya E., The investigation of the structural properties of 3-nitrophthalonitrile using spectroscopic and quantum chemical computational methods, Int. J. Comput. Exp. Sci. Eng., 2017, 3(2), 37-40.

[15] Ditchfield R., Molecular orbital theory of magnetic shielding and magnetic susceptibility, J. Chem. Phys., 1972, 56(11), 56885691.
[16] Woliński K.., Hinton J.F., Pulay P., Efficient implementation of the gauge-independent atomic orbital method for NMR chemical shift calculations, J. Am. Chem. Soc., 1990, 112(23), 8251-8260.

[17] Balci K., Akyuz S., A vibrational spectroscopic investigation on benzocaine molecule, Vib. Spectrosc., 2008, 48(2), 215-228.

[18] Sundaraganesan N., Ilakiamani S., Saleem H., Wojciechowski P.M., Michalska D., FT-Raman and FT-IR spectra, vibrational assignments and density functional studies of 5-bromo2-nitropyridine, Spectrochim. Acta, Part A, 2005, 61(13-14), 2995-3001.

[19] Fukui K., Role of frontier orbitals in chemical reactions, Science, 1982, 218, 747-754.

[20] Pearson R.G., Absolute electronegativity and hardness correlated with molecular orbital theory, P. Natl. Acad. Sci. USA, 1986, 83, 8440-8441.

[21] Lee J., Hoon K., Moon J.P., Long-life, high-rate lithium-organic batteries based on naphthoquinone derivatives, Chem. Mater., 2016, 28(7), 2408-2416.

[22] Geetha K., Umadevi M., Sathe G.V., Vanelle P., Terme T., Khoumeri O., Surface enhanced Raman spectral studies of 2-bromo-1,4-naphthoquinone, Spectrochim. Acta, Part A, 2015, 138, 113-119.

[23] Ebrahimipour S.Y., Sheikhshoaie I., Crochet A., Khaleghi M., Fromm K.M., A new mixed-ligand copper(II) complex of (E)- $\mathrm{N}^{\prime}$-(2-hydroxybenzylidene) acetohydrazide: Synthesis, characterization, NLO behavior, DFT calculation and biological activities, J. Mol. Struct., 2014, 1072, 267-276. 